FROM BIM TO VR: DEFINING A LEVEL OF DETAIL TO GUIDE VIRTUAL REALITY NARRATIVES

\title{
SPECIAL ISSUE: Virtual, Augmented and Mixed: New Realities in Construction
}

PUBLISHED: December 2019 at https://www.itcon.org/2019/31

EDITORS: McMeel D. \& Gonzalez V. A.

DOI: $10.36680 /$ j.itcon.2019.031

\section{Katie Graham}

Instructor, Media Production and Design, School of Journalism and Communication, Carleton University Associate Faculty, Carleton Immersive Media Studio, Carleton University

katelyngraham@cunet.carleton.ca

Lara Chow, Associate Director

Carleton Immersive Media Studio, Carleton University, https://www.cims.carleton.ca

Lchow@cims.carleton.ca

\author{
Stephen Fai, Director and Associate Professor \\ Carleton Immersive Media Studio, Carleton University, https://www.cims.carleton.ca \\ sfai@cims.carleton.ca
}

SUMMARY: In 2012, the Carleton Immersive Media Studio (CIMS) started a research relationship with Public Services and Procurement Canada to develop a building information model (BIM) of the Parliament Hill National Historic Site of Canada. The model was created to facilitate a multi-year rehabilitation of the site and was developed using both historical records and highly detailed geo-referenced point cloud data. In the process of planning the model, CIMS developed a unique Level of Detail (LOD) specification for heritage buildings that, in addition to standard specifications, considered cultural heritage value as part of the LOD. As the rehabilitation project unfolded, the possibility of using the BIM for public engagement through the creation of virtual reality (VR) experiences was proposed. In this paper, we discuss the transferal of CIMS' LOD from a BIM to a VR environment, arguing that the BIM LOD's focus on cultural heritage value is consistent with virtual reality LOD in that it can be used to guide participants through a virtual reality narrative by inferring that areas of higher fidelity have greater value.

KEYWORDS: Virtual Reality, Building Information Modelling, Level of Detail, Narrative, Storytelling

REFERENCE: Katie Graham, Lara Chow, Stephen Fai (2019). From BIM to VR: defining a level of detail to guide virtual reality narratives. Journal of Information Technology in Construction (ITcon), Special issue: 'Virtual, Augmented and Mixed: New Realities in Construction', Vol. 24, pg. 553-568, DOI: 10.36680/j.itcon.2019.031

COPYRIGHT: () 2019 The author(s). This is an open access article distributed under the terms of the Creative Commons Attribution 4.0 International (https://creativecommons.org/licenses/by/4.0/), which permits unrestricted use, distribution, and reproduction in any medium, provided the original work is properly cited.

\section{cc)creative}




\section{INTRODUCTION}

In 2019, the Centre Block of Parliament Hill National Historic Site of Canada closed to the public for a multi-year rehabilitation project. Since 2013, the Carleton Immersive Media Studio (CIMS), a research unit associated with the Azrieli School of Architecture and Urbanism at Carleton University in Ottawa, Canada, has had the opportunity to be involved in the digitization and modelling of the existing conditions of the site to prepare for the rehabilitation program of work. CIMS is now helping to leverage this data for public engagement through various digitally assisted storytelling projects — including active and passive virtual reality (VR). While developing VR protocols to manage the quality of assets, maintain optimal functionality, and control file size for public dissemination, CIMS reflected on our past work in developing a unique Level of Detail system (LOD) for heritage BIM which brought similar challenges and benefits.

During the development of the Parliament Hill BIMs, Level of Detail guidlines and standards for heritage buildings did not exist. To establish consistency in our work, CIMS began the development of internal LOD guidelines in 2015 called CIMS LODIA. The three-tier, five category system borrows from the architecture, engineering, construction and operations (AECO) industry standards and guidelines and indexes level of detail (LOD), level of information (LOI), and level of accuracy (LOA) — LODIA — for each element type (Graham et al., 2018). As the model development progressed — and subsequently our expertise for developing modelling workflows for complex building assets - our research uncovered a correlation between LOD, BIM use, and cultural heritage value. Generally, the higher the LOD for assets, the higher the significance of cultural heritage. This focus on the cultural heritage value within the LOD classification proved to also have merit when translating to VR, helping to indicate which elements within the environment should be of higher detail. When transferring this LOD schema to VR, the assets within the virtual experience modelled at a high LOD are those important to the narrative of the story and are understood to have heritage value. In turn, CIMS created a classification system for the LOD within VR to help identify which spaces and assets are of value for the heritage narrative in the VR environment.

This paper will introduce the main concepts of CIMS LODIA and will demonstrate a relationship between LOD, BIM use, and cultural heritage value and how this is translated into a LOD classification for heritage spaces in VR. While comparing how commonly used LOD systems for new construction and computer graphics prove insufficient for the representation of architectural heritage, a new methodology for organizing architectural heritage spaces in VR will be introduced. Using our work on the Parliament Hill National Historic Site, we will provide examples that demonstrate the effectiveness of the LOD system for heritage spaces in VR - both in helping to define a narrative and in evoking the cultural value of a space, while maintaining optimal performance through file size management.

\section{THE REHABILITATION PROJECT OF THE PARLIAMENT HILL NATIONAL HISTORIC SITE}

Construction of the Parliament Hill National Historic Site - comprised of the Library of Parliament, Centre Block, West Block, East Block, and grounds - began in 1859. It quickly became a symbol of Canadian parliamentary democracy and admired for its exemplary Gothic Revival style. In 1916, a fire destroyed the original Centre Block building. A ten-year reconstruction began in 1917 following the new design of John A. Pearson and Jean Omer Marchand. The new building showcased cutting edge technologies for construction at the time - such as a new steel structural system - while retaining the ornamental gothic revival quality of the other buildings still standing within the campus.

In 2002, a comprehensive rehabilitation project began on Parliament Hill to repair the historic fabric, modernize services, and address changes in its functional program. To date, two buildings have been completed the Library of Parliament (2007) and the West Block (2018). The Centre Block program of work began in 2018, and the East Block will follow shortly thereafter. During the significant rehabilitation program, tours of the Centre Block will be suspended, with alternate means of engaging with the public, both on-site and remotely, being explored. This includes virtual reality and other digitally assisted storytelling methods. 


\subsection{The digitization and modelling by CIMS}

In 2012, Public Services and Procurement Canada (PSPC) and CIMS began a research partnership to explore the application of digital technologies for architectural rehabilitation and heritage conservation. Research focused on the Parliament Buildings National Historic Site of Canada and explored methodologies for digitization (integrating photogrammetry and terrestrial laser scanning); building information modelling of historic structures (HBIM); digitally assisted fabrication (robotic milling and 3D printing); and digitally assisted storytelling (web, mobile, and virtual and augmented reality).

Digitization and BIM research on Parliament Hill began with the West Block BIM project (2013), leading to the Centre Block BIM project (2015), and culminated in the Library of Parliament BIM project (2017) (Figure 1). The models were created in Autodesk Revit from diverse data sets with the primary source being georeferenced point cloud data. Due to the accuracy of the metric information and the capacity to capture material deviation in the building elements, the primary data source was geo-referenced point cloud data from terrestrial laser scanning and photogrammetry. The terrestrial laser scanning was completed by CIMS in partnership with Heritage Conservation Services (HCS) - a branch of Public Services and Procurement Canada - using a Leica C10 and P40 (exterior and large interior spaces) and a Faro Focus (small to mid-sized interior spaces). Over 2000 individual scan stations were required to capture the interior and exterior of Centre Block - resulting in over four terabytes of point cloud data. In addition to terrestrial laser scanning, significant heritage interiors including the Senate, Senate Foyer, House of Commons, House of Commons Foyer, Rotunda, Hall of Honour and the exterior of the Peace Tower were captured by HCS using photogrammetry and 360 photography.

Secondary sources such as archival drawings, historical photographs, historical steel catalogues, and technical reports were referenced in cases where point cloud data was not available. For example, structural steel catalogues were referenced since the structural steel is normally hidden from view and cannot be captured by laser scanning or photogrammetry.

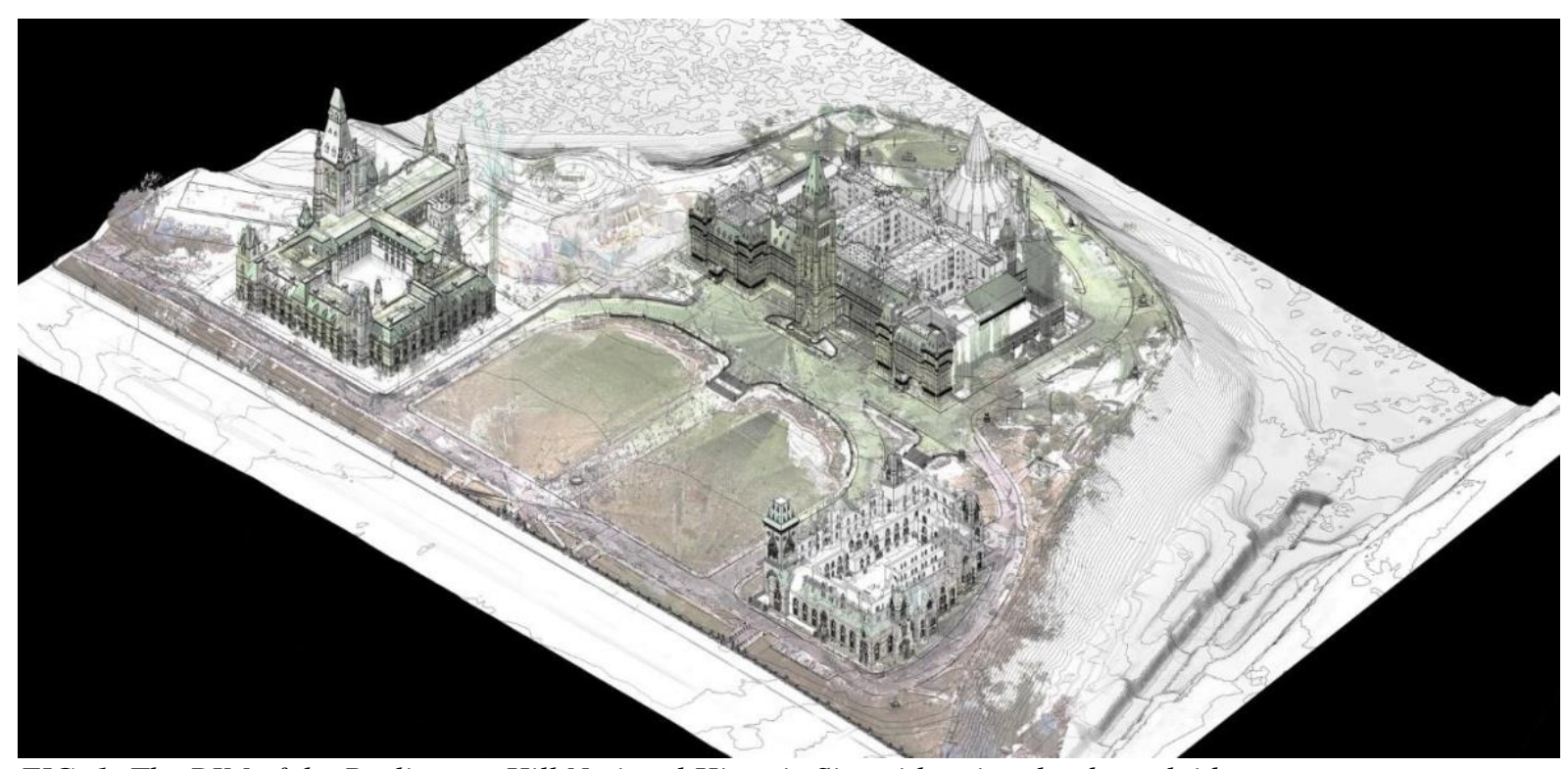

FIG. 1: The BIM of the Parliament Hill National Historic Site with point cloud overlaid.

In addition to capturing the existing conditions of the building, the model was developed in anticipation of specific model uses including, but not limited to, the generation of drawings, site analysis, design coordination, design authoring, and heritage asset management.

For the Centre Block BIM, the initial proposal was to utilize a commonly accepted BIM specification classification system - The Level of Development Specification 2015 developed in the United States by BIMFORUM (BIMFORUM, 2015). The system combines level of detail with level of information classifications into 'Level of Development.' A simplified level of development system was established between PSPC and CIMS, assigning Level of Development to specific building element categories: LOD 300: exterior walls, roofs, foundations, structural elements (verified to point cloud); LOD 200: interior walls, stairways, slabs, structural elements (not 
verified to point cloud). LOD 300 was also assigned to spaces of significant heritage value such as the Senate Chamber, House of Commons chambers, Senate and House of Commons foyers, Rotunda, and Hall of Honour. For example, according to The Level of Development Specification 2015, an exterior masonry wall at LOD 200 will be modelled as a generic wall object separated by type of material and have flexible layouts and locations, while at LOD 300 the model element will contain additional metadata such as member size and design loads at a design-specified location.

As modelling progressed, it become evident that the BIMFORUM specification was insufficient for developing BIMs of historical buildings such as Centre Block. The availability of information for in-situ building elements varied significantly, creating the need to index levels of geometric detail, non-graphical information, and accuracy for each model element type. Unlike BIM for new construction, where Level of Detail and Level of Information increase as the project moves from concept through construction - the starting point for HBIM begins with the collection of all as-built and as-found metric and graphic information available which can result in a disproportional relationship between LOD and LOI. To clarify the terms of reference, CIMS proposed the use of the CIMS LODIA - resulting in a more nuanced system of classification, discussed in further detail below. CIMS LODIA breaks the specification into three distinct categories - detail, information, and accuracy.

\section{DEVELOPMENT HERITAGE LOD GUIDELINES}

\subsection{Level of Detail/Development Systems for BIM}

In the Architecture, Engineering, Construction, and Operations (AECO) industry, BIM is intended as a multidisciplinary communications tool for stakeholders and therefore needs to follow guidelines or standards like a Level of Detail or Development classification system — to maintain consistent language among stakeholders. Several international BIM organizations have developed guidelines, such as The Level of Development Specification (BIMFORUM, 2015) created by BIMFORUM in the United States and PAS 11922013 (BSI Group, 2013), developed in the United Kingdom by the British Standards Institution (BSI). These standards are created primarily for new construction and focus on LOD and LOI requirements for different phases of a new-build project such as concept, design, construction, and operation — with little or no discussion of asfound or heritage modelling.

These existing guidelines for new construction hold commonalities between them such as the progression from a simplified placeholder object at the lowest LOD to a graphically detailed object with all related information at the highest LOD. The classification systems define both the graphical representation and embedded information related to the representation; however, it is not possible to accommodate the disparity between geometric information and non-geometric information — commonly found within existing buildings — using these systems.

Although the application of and literature on BIM for heritage buildings has been increasing rapidly, one of the fundamental problems, as outlined in several literature reviews, is the lack of consensus on HBIM standards and classifications (Dore, 2017; Hichri et al., 2013; Volk et al., 2014). To date, only two guides have been published addressing the need for BIM specifications for historic buildings. Specifically, Historic England's BIM for Heritage (Antonopoulou and Bryan, 2017) and Plowman Craven's BIM Survey Specification and Reference Guide (Plowman Craven, 2015). These guides build upon existing new construction classifications systems for LOD, LOI and LOA and augment these systems for historical and heritage BIM.

\subsection{CIMS Level of Detail/ Information / Accuracy (LODIA)}

In 2015, CIMS began developing internal LOD guidelines to address the lack of BIM guidelines or standards for existing and heritage buildings. The three-tier, five category level of development system borrows from AEC industry standards and guidelines and maps level of detail (LOD), level of information (LOI), and level of accuracy /modelling tolerance (LOA)_LODIA — for each element type.

The level of detail (LOD) describes the graphical representation of the model and follows the existing standards referenced from symbolic placeholder to detailed model in the categories LOD 0 to LOD 4 (Figure 2). The choice of a specific level of detail is determined by available reference material and the purpose of the model. 


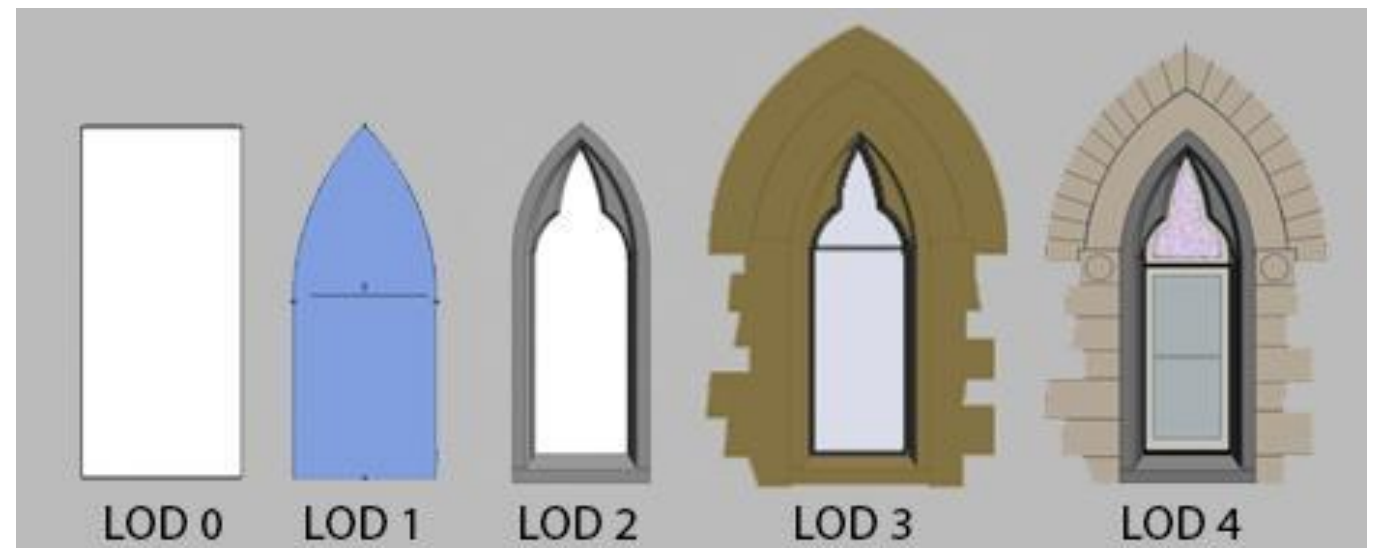

FIG. 2: CIMS Level of Detail 0 to 4 of a window

The level of information (LOI) is the embedded information that BIM elements contain such as material, manufacturer, source data, and additional resources. The level of information is often determined by the data available. Unlike a new-build — where the material and assembly of a wall are known - a BIM of an existing or heritage building relies on existing drawings, specification documents, and on-site investigation to determine structure, material, etc. In some cases, only on-site documentation and observation are available.

The level of accuracy (LOA), or modelling tolerance, refers to how closely the BIM fits against the point cloud data. In the context of the CIMS LODIA protocol, LOA determines the acceptable level of modelling tolerance expressed as a +/-mm figure. For the Centre Block BIM, a model tolerance of $+/-25 \mathrm{~mm}$ was agreed upon with any building vertical or horizontal greater than $25 \mathrm{~mm}$ captured in the model element. Any horizontal deviations, vertical deviations, and / or irregularities in geometry were also identified in the properties panel of the model element through a custom text parameter.

The next phase of our research was to study the possible adaptation of CIMS LODIA for virtual reality. While all of the specialty rooms in the Centre Block BIM were initially targeted to be modelled at a LOD 300 from the BIMFORUM guide, they were increased in graphical detail to a LOD 4 and LOA 4, while LOI was reduced to 2 (Figure 3). This was because the model geometry was to be leveraged through new BIM uses that required a high LOD such as heritage asset management, visualizations, and a virtual reality project related to the rehabilitation.

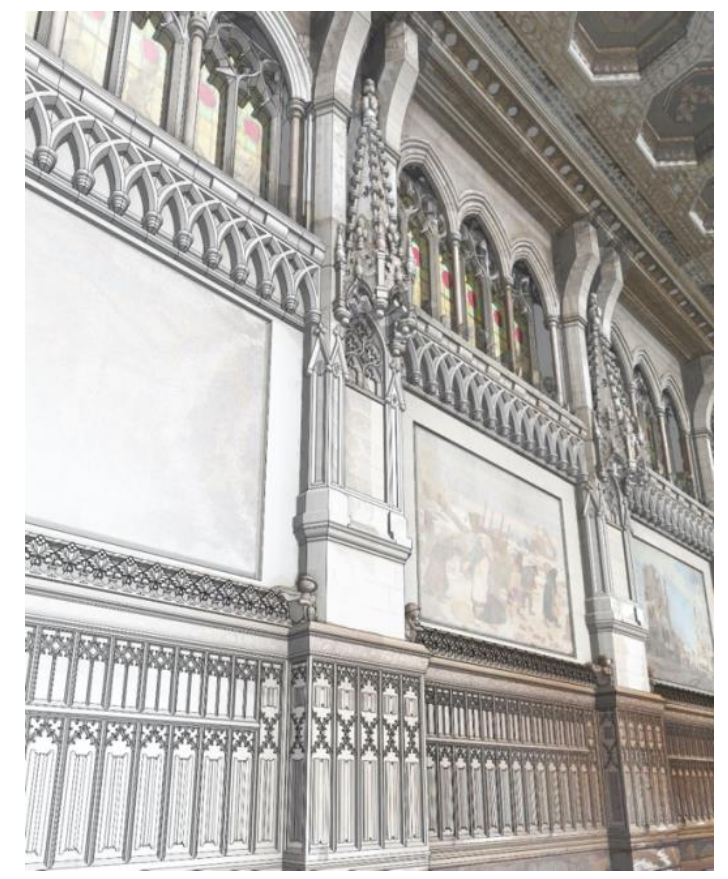

FIG. 3: Image of BIM of the Senate Chamber showing the high level of detail. 


\section{DISSEMINATION THROUGH DIGITALLY ASSISTED STORYTELLING}

Working with the Parliamentary Precinct Branch (PPB) and others, CIMS has had the opportunity to leverage the digital recording and modelling of the Parliament Hill National Historic Site for digitally assisted narratives directed at partner and public engagement with the rehabilitation project. These narratives have been developed for a wide range of media including web, mobile, in-situ, and passive and active VR experiences. The first passive VR, in the form of 360 video experiences, was released in 2017 through an on-site installation — the VR Kiosk (Figure 4) - in Ottawa, Canada.

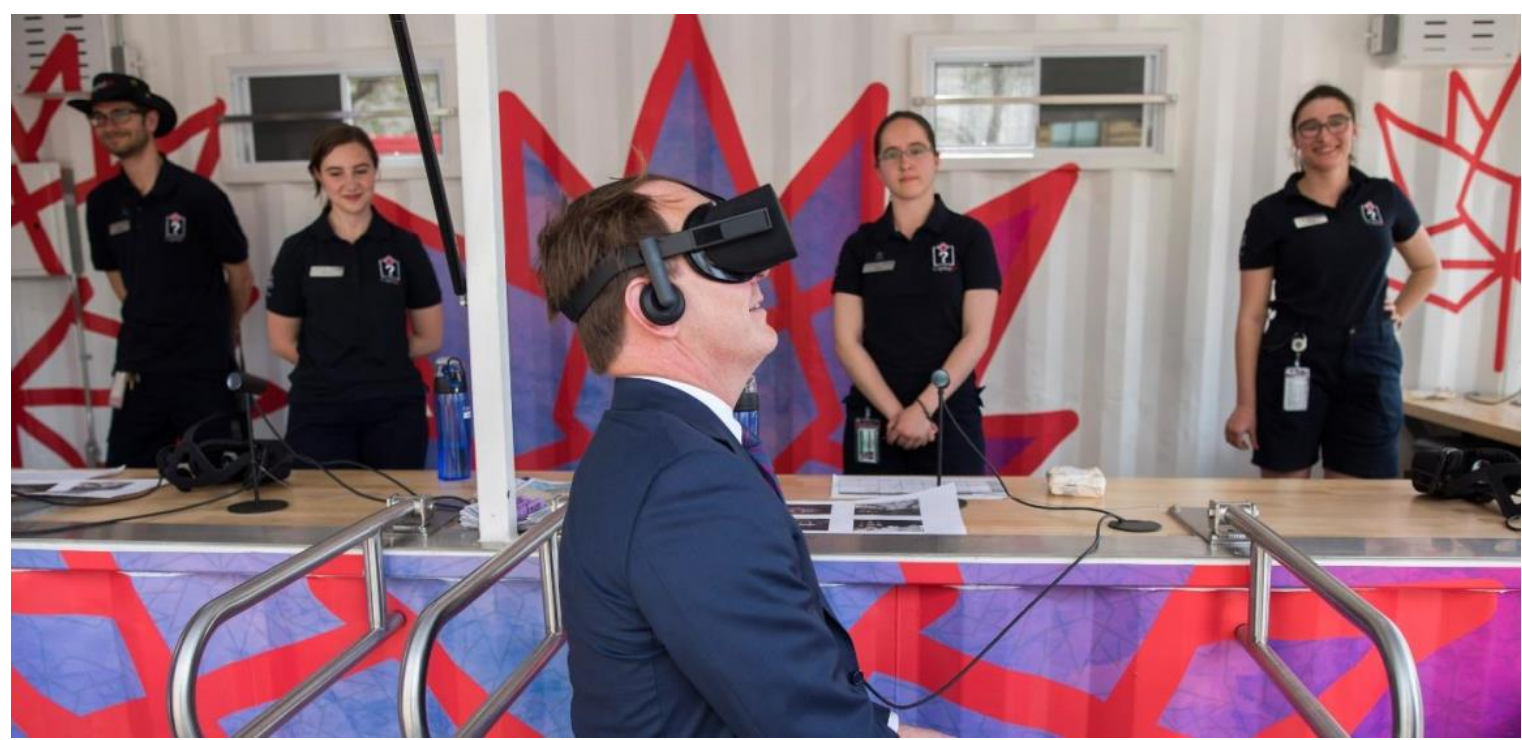

FIG. 4: The VR Kiosk at the Capital Information Kiosk across from Parliament Hill allowed visitors to explore passive VR. Photo by Chris Roussakis.

Within the passive 360 video experiences of the VR Kiosk, visual assets included a combination of panoramic photography, point cloud data, BIM, and historical photos to create a hybrid visualization. The experiences were processed using Adobe Premiere and Adobe After Effects to create a 360 video to be experienced on an Oculus Rift VR headset. Careful selection of visual stimuli was made and overlaid with a soundtrack and narrative that helped to draw the viewer's attention to the desired elements of the scene. The storylines within the 360 video experiences were linear with the careful control of what was visible and how the story unfolded.

CIMS research in active VR using game engine technology began in 2018. Active VR is best described to have a high degree of presence - the feeling of 'being there' - and a strong sense of agency - the feeling of control over the experience. Presence, a term first introduced as telepresence by Marvin Minsky in relation to teleoperations and the control of remote robots (Minsky, 1980), was later translated to describe the feeling and belief of being present in a virtual world (Held and Durlach, 1992; Sheridan, 1992; Slater and Usoh, 1993; Steuer, 1992; Zeltzer, 1992). According to researchers, presence is achieved when the virtual world behaves consistently, avoids interference from external elements, and contains a correlation between the user's physical and virtual movements. Agency, or the ability to influence the environment and narrative (Murray, 1997), will affect the sense of presence by providing the connection between physical actions and virtual outcomes (Slater et al., 1994).

This enhanced sense of presence and agency comes with increasing demands on hardware, software, and narrative development. In the VR Kiosk, the viewer remains stationary while the scene unfolds around them. As exported video files, the experiences of the VR Kiosk do not require real-time rendering or put a high demand on computer software and hardware. Within the passive experiences, the narrative is controlled by the linear flow of the video and the reduced agency of the user. The active experiences, however, allow for an increased agency, and therefore, less control of the narrative outcome. When the goal is to provide a concise narrative within the experience, methods to control where the user's attention is drawn should be implemented to help control the narrative. CIMS hypothesized that a LOD system that emphasized the elements with cultural heritage value and in the space would help attract the user's attention. CIMS developed the new LOD system for virtual reality, borrowing from the internal classification system for BIM - LODIA - that defines the architectural elements of high heritage significance to be of higher fidelity than less essential elements. 


\section{CREATING AN LOD FOR VIRTUAL REALITY HERITAGE SPACES}

\subsection{Existing LOD Systems for Computer Graphics}

While LOD systems for digital spaces are well established, they lack any attempt at an industry-standard related to content. Instead, they are typically used to optimize polygon count and processor speed. First introduced in 1976 by James H. Clark in Hierarchical Geometric Models for Visible Surface Algorithms, the guidelines proposed that objects farther away from the camera should be at a lower level of detail than those closer to the camera. As Clark explains, the eyes of a human figure in the distance would require minimal representation whereas seen close up would require more detail. What can be represented as a black speck at one distance must have a visible distinction between iris and retina at another. By looking at the proximity of objects within a scene as a hierarchy and modelling only "...the minimal information needed to convey the meaning of what is being viewed..." (Clark, 1976), the geometric complexity and polygon count of the scene can be carefully maintained to meet industry standards.

This concept of proximity-based LOD was used in developing the passive VR experiences of the VR Kiosk. Objects in the distance were simplified while those in the foreground were rendered at a higher resolution. For example, in the experience, From Yesterday to Today: Discover the Hill, lower resolution historic photography comprised the buildings in the distance, while the camera was situated on a more detailed terrain model with highresolution images populating the scene between the viewer and the background (Figure 5). Essentially, object resolution was dictated by the distance from the camera.

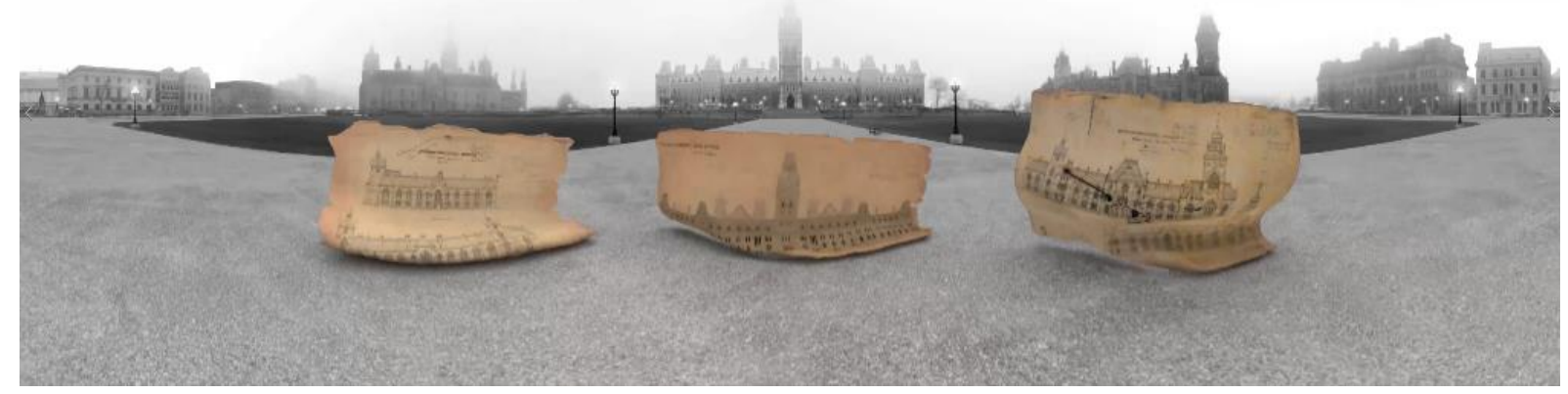

FIG. 5: Screenshot from the VR Kiosk experience, From Yesterday to Today: Discover the Hill.

Unlike the passive scenes of the VR Kiosk - where objects are rendered at chosen LODs and placed in relation to a fixed camera - typical scenes in video games and active virtual experiences allow the participant and objects to move around, creating the need for the objects of the scene to change between different LODs relative to proximity. Three common frameworks are currently used to identify LODs within a scene: discrete, continuous, and view-dependent.

The Discrete LOD framework is the method that Clark proposed in his 1976 paper. Multiple versions of an object are created at predefined LODs. During the real-time play of the experience, the different versions are loaded is based on the proximity of the participant to the object (Luebke, 2003). For example, if the asset is a sphere, from a distance, it will be modelled as the simplified dodecahedron. As the object gets closer, through the movement of the participant or the scene, the digital form will be replaced with more elaborate shapes until it appears as the poly-heavy sphere (Clark, 1976).

Continuous LOD, also known as Progressive LOD, creates a complex data structure that contains multiple LODs within it. During run-time, the appropriate LOD is pulled out, and additional details are left unloaded. This framework creates better granularity and fidelity due to the smooth transition between LODs pulled from one source and not dictated by a predetermined set (Luebke, 2003).

The final LOD framework, View-Dependent LOD, allows for an object to be simplified anisotropically relative to the distance or point of view of the camera. For example, a large field, such as a landscape, will have the portion 
currently being viewed by the camera shown at a high LOD while the distant edges would be modelled with less detail. Similarly, only the portion of an object being viewed — for instance, an object with a front and back will only be partially visible, allowing the back — or the side not currently in the camera — to be reduced (Luebke, 2003).

The LOD systems in computer graphics have not been systematically developed for the development of narrative content and are primarily a method for communicating degrees of modelling complexity in a virtual scene to minimize polygon count and optimize processing speed. These existing models proved inadequate for creating a VR space that would use the modelled assets as a method to highlight elements of cultural heritage value. The existing methods could be used in conjunction with the proposed model to help further reduce the performance demand on computer hardware and software. Within the development of the VR experience of the Centre Block, CIMS employed the proximity-based LOD in addition to the new method. The choice to exclude the discrete, continuous, and view-dependent LOD systems was made due to scope and resource limitations. Duplicate modelling to create lower-resolution duplicate meshes would add considerable time as well as add additional objects to require loading within Unity 3D. The decision was made to only use proximity-based LOD in addition to the new method since the VR experience in development would not allow teleportation - allowing objects that remain outside a small playable space to stay unreachable.

When creating active virtual reality experiences of heritage spaces, the essential assets related to heritage value or narrative of the space must first be identified. Although the computer graphics LOD systems are helpful when relating model complexity to proximity and to optimize processor speed, for heritage spaces, certain assets in the room are integral to the overall cultural heritage value of the space while others are not. A VR LOD classification for a heritage space should reflect both the proximity criteria of existing standards and the cultural heritage value to aid in the overall narrative of the space.

\subsection{Lessons Learned from the CIMS LODIA}

To help define a VR LOD for heritage spaces, we considered the lessons learned from the creation of CIMS LODIA for BIM. The LOD within the BIM classification system is linked to the elements of cultural heritage value due to specific BIM uses such as the desire to show important elements at a high visual representation. For the virtual reality experience, assets within the spaces that are of minimal historical and cultural relevance are either omitted or represented at the lowest LOD since they will not be needed for visualizations, whereas assets of high heritage value are represented at the highest LOD. For example, within the Centre Block of Parliament Hill BIM, the hallways and attic space are modelled according to LODIA at LOD2 while the Senate Foyer is modelled at a LOD4 (Figure 6).

An effort was placed in ensuring the stone detailing and complex ceiling structure within the Senate Foyer were modelled while the hallway and attic were left with minimal graphical representation. Within the BIM, the level of heritage and cultural importance of assets were determined by a heritage asset database with over 15,000 assets created by PSPC. For the VR LOD's use in the Centre Block, the significance of the assets was also determined by a review of the spaces by all project stakeholders. Elements that helped define the architectural space or were critical to the narrative of the VR experience were given high value.

The LOI and LOA within the BIM classification system both dealt with elements relating more to the construction management of the project and were deemed not relevant for visualizations in VR. The embedded information of material, reference documents, and verification status that defined the LOI were critical for the rehabilitation project but not for all the different purposes of the model. The LOA helped for accuracy in construction planning and had a minor visual effect on the model; however, it proved to impede/limit the optimization of the VR space. In areas modelled in a high LOA, each minor variation between identical window type is recorded, creating unique window meshes. To optimize the model for VR, the LOA was discounted, and identical window families were represented with a generalized mesh that disregarded small differences in proportions. This process allows for only one geometry to be loaded within the space and then repeated, reducing the processing time of the experience. For most elements, the ideal condition found in the repeated elements will not affect the heritage aesthetic of the environment; however, if the narrative requires attention to the variances, the unique geometry will need to be used. 


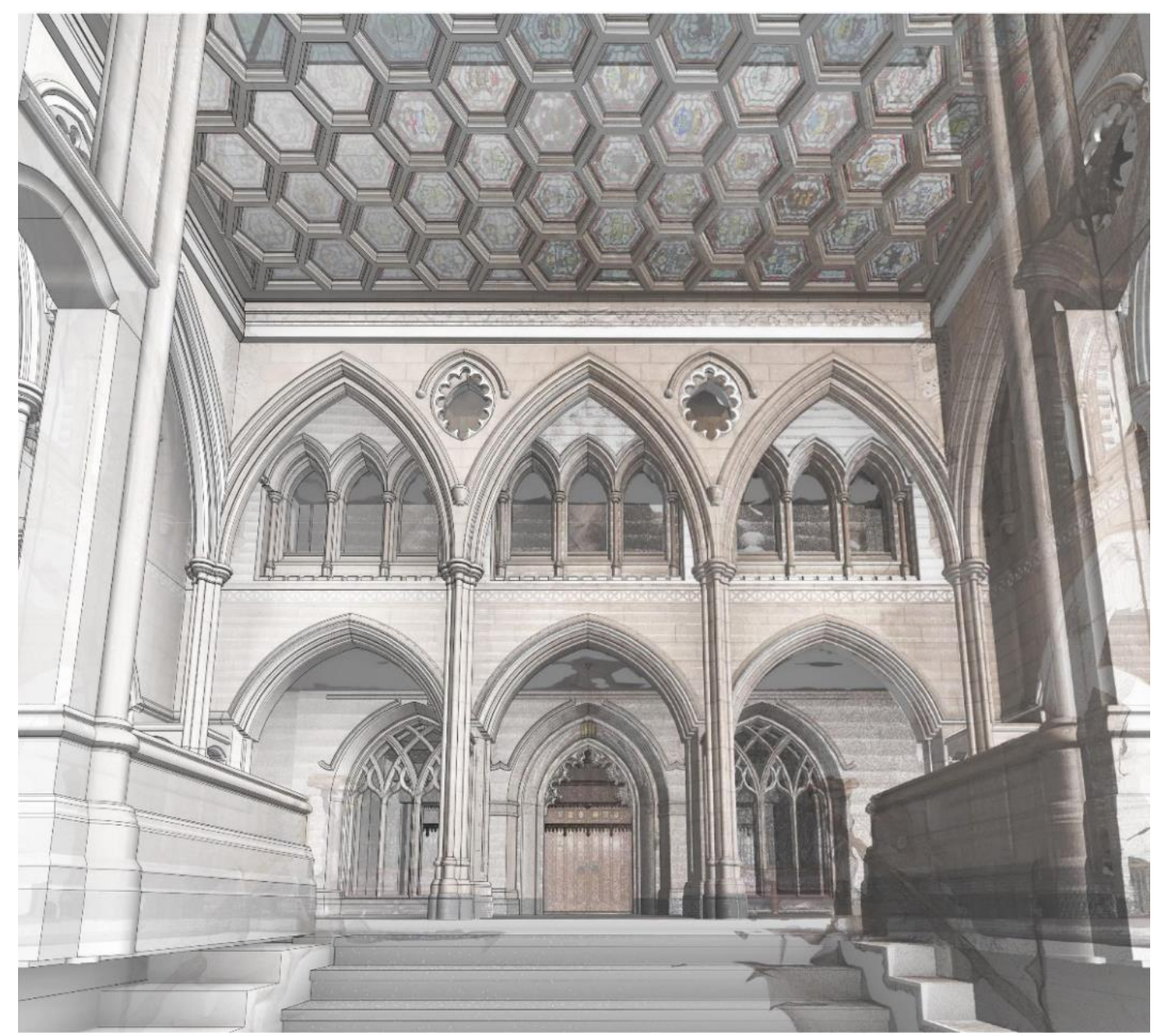

FIG. 6: The Senate Foyer modelled at LOD4, according to LODIA.

\subsection{Translation Process from BIM to Virtual Reality}

CIMS converted the detailed BIM into a VR experience that could be explored through the game engine Unity 3D and the Oculus Rift VR headset. Although the BIM contained a level of detail appropriate for visualizations and renderings, the data was not easily converted into Unity 3D for VR due to the complexity of geometry, unoptimized meshes, and unrealistic textures. CIMS devised a workflow using Rhinoceros 3D and Autodesk 3DS Max to help convert the BIM into a working VR experience. In developing this workflow, the criteria outlined by Oculus Rift — for optimized performance of two-million maximum polycount per VR model (Oculus Documentation, n.d.) served as an important guideline.

Within Autodesk Revit, the geometry associated with the desired space is isolated and exported as a DWG file and imported into Rhinoceros 3D. The model is converted into a mesh, correcting import errors, and organizing repeated elements into block instances to reduce overall unique geometry. The mesh is exported in a Collada format to preserve the block instanced geometry and imported into Autodesk 3DS Max to correct UV mapping errors and to produce required normal maps for the appearance of detailed textures. The meshes are exported as FBX files and imported into Unity3D where proper textures are applied. In the case of meshes where the texture is derived from the point cloud data, photogrammetry, or high-resolution photographs, the source files are processed using InstaLOD and Adobe Photoshop. This conversion process is outlined in more detail in a separate publication (Pybus et al., 2019).

Meshes developed using photogrammetry (PhotoScan) were used within the model for areas of high LOD. These meshes were optimized using InstaLOD, to reduce the overall polycount and create normal and UV maps. The software allowed for the reduction of the polycount by an average of 20 times. For example, the Queen Victoria Statue in the Library of Parliament that was reduced from 198,587 polygons to 8,078 (Figure 7). 


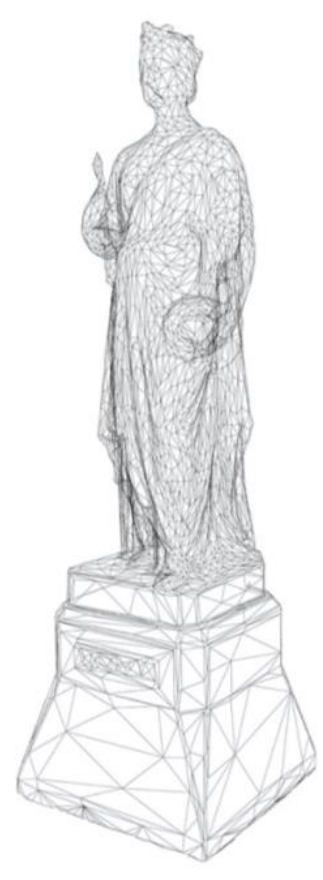

Low Triangulation
Polygon Count: 8,078

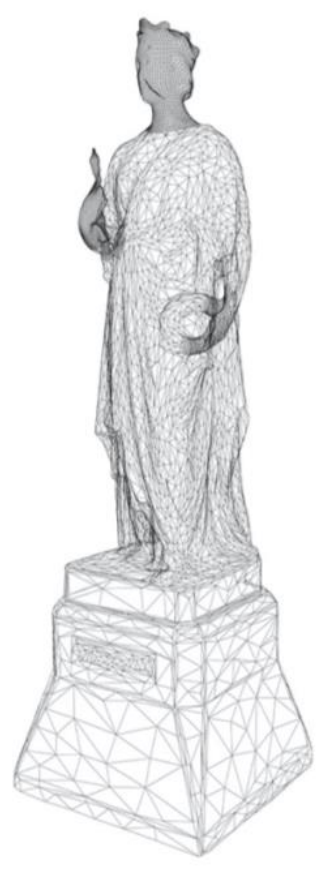

Medium Triangulation
Polygon Count: 24,233

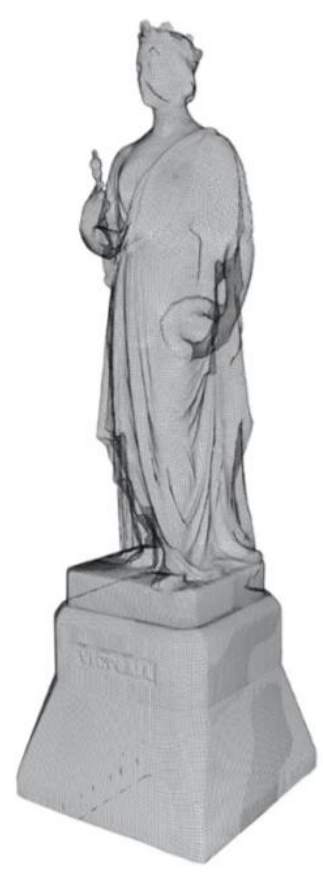

High Triangulation
Polygon Count: 198.587

FIG. 7: Queen Victoria statue showing levels of reduction in polycount.

\subsection{LOD VR Classification for Heritage Spaces}

The LOD VR classification system (Figure 8) for heritage spaces that CIMS has developed uses a three-tier system that focuses on the heritage and cultural significance of assets. On the low end of the spectrum is LOD 1 - with a clean mesh that uses normal maps for complex geometries and generic texturing. LOD 1.5 expands on LOD 1 by texturing the model with photogrammetry, photography, or point cloud data. LOD 2 replaces the built mesh with an optimized photogrammetric model to ensure all details are represented and the heritage asset is highlighted. LOD 2 is intended only to be used for assets of high importance and interest due to their high polygon count. Below is a detailed breakdown of each LOD.
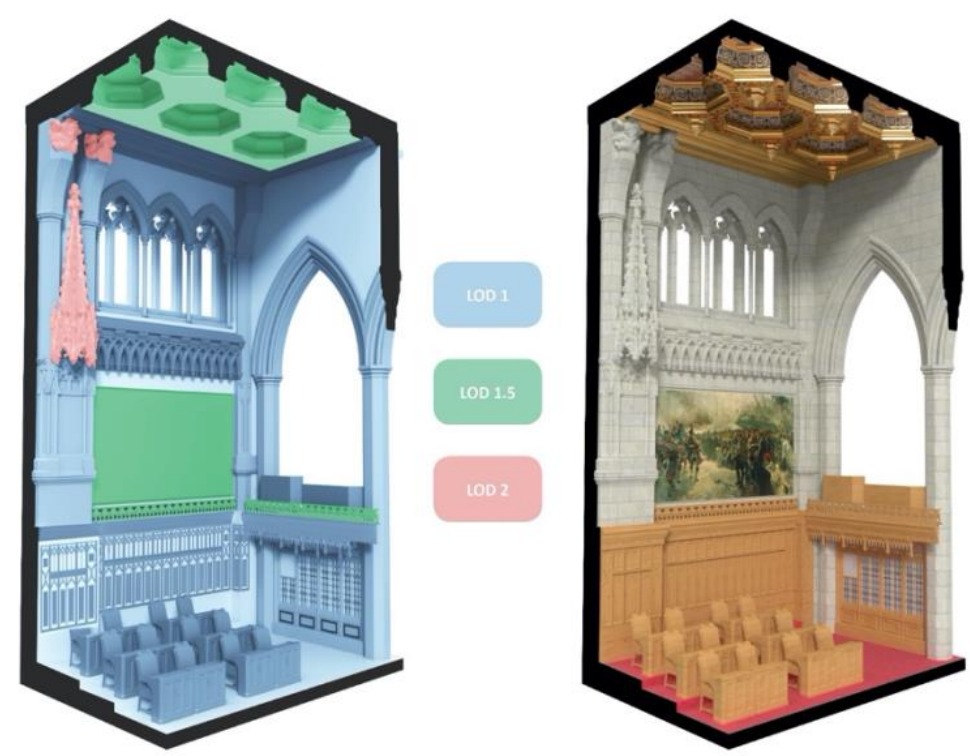

Figure 8: Illustration showing the LOD 1, LOD 1.5, and LOD 2 in a portion of the Senate Chamber. 


\subsubsection{LOD 1}

The lowest LOD classification is LOD 1, which consists of a mesh with generic textures. In the CIMS workflow, the mesh is created by converting a LOD4 BIM model into an optimized mesh using the computer modelling software Rhinoceros 3D. The use of the BIM to create the virtual reality assets takes advantage of the digital assets CIMS has previously created as well as ensuring an accurate representation of the space. Textures from a generic texture library can be applied to give the assets a sense of materiality; however, the textures are not custom or unique to the asset. The LOD 1 assets help give context to the heritage space and provide a backdrop to more significant components without drawing attention to themselves.

Within the spaces converted into VR, the majority of assets are modelled at an LOD 1. Within the Senate Chamber, the stone walls, flooring, wood panelling, doorways, and a majority of the furniture does not contain as high of cultural heritage value as the other assets, such as the stone canopies, ornate ceiling, and sculptures above the Speaker's chair. To create the LOD 1 elements, they are modelled in Autodesk Revit and imported into Rhinoceros and Autodesk 3Ds Max for conversion into a Unity 3D compatible mesh. The finished product can be seen in the desks of the Senate Chamber (Figure 9).

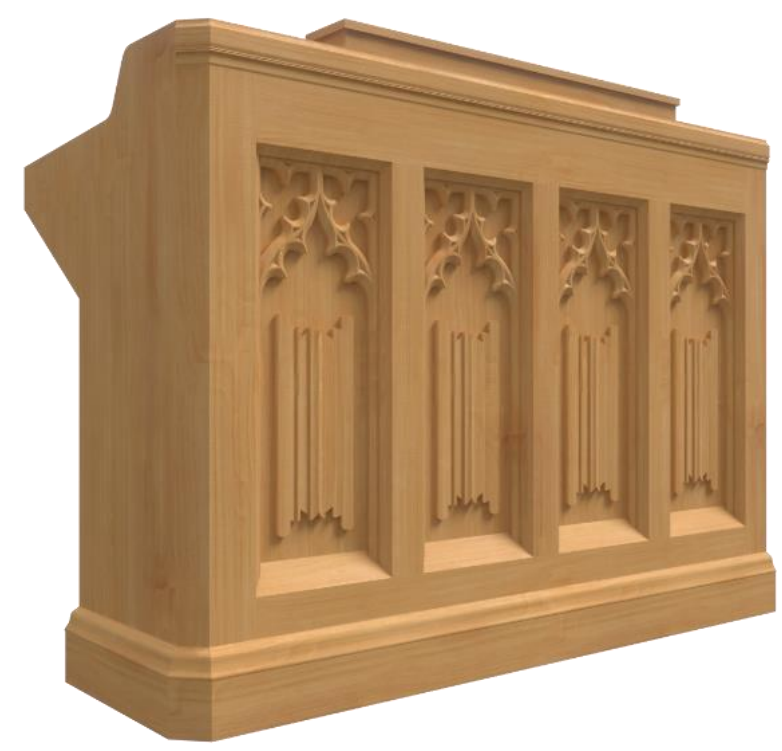

FIG. 9: The desks in the Senate Chamber are an example of LOD 1.

\subsubsection{LOD 1.5}

LOD 1.5 uses the LOD 1 mesh but has a non-generic, site-specific texture. Point cloud data from terrestrial laser scanning, photogrammetry, or photography can be used to help generate textures for LOD 1.5 assets. The polygon count of the mesh remains equal to LOD 1 with any additional visible detail appearing as a property of the texture. The quality of the LOD 1.5 model is dependent on the quality of the colour data and requires additional time to acquire, process, and convert into a viable texture. Considerable effort is required to capture and edit the photographic data to properly match the mesh. Although LOD 1.5 assets do not increase the polygon count and do not effect optimization of the scene, it is a large time commitment that may not be warranted for all elements. LOD 1.5 is best used on areas that have high cultural heritage value but do not require geometric complexity. Ceilings, floors, and walls with complex painting or relief are examples.

Within the Senate Chamber, the ceiling (Figure 10) was created at LOD 1.5. The ceiling is modelled in Autodesk Revit and converted into a Unity 3D compatible mesh using Rhinoceros and Autodesk 3Ds Max. The point cloud data of the ceiling is converted into an orthographic projection image that is processed in Adobe Photoshop. Elements, such as the crests and unique textures, are isolated and applied as individual textures to the mesh. The isolation of the small elements is crucial to ensure that the photographic data carefully matches the geometry of the mesh, that through optimization may have been altered to a more generalized form. The ceiling contains a higher level of detail than the contrasting LOD 1 elements, drawing the visitor's attention to it - while maintaining a lower polycount than an equivalent LOD 2 element. 


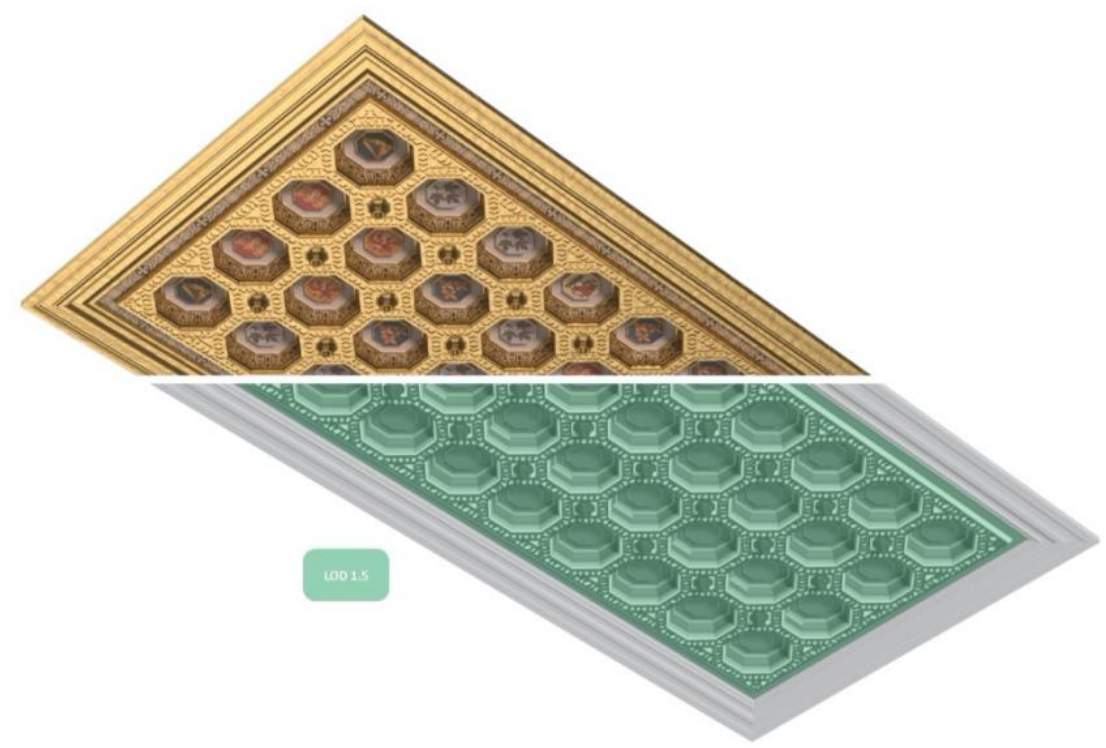

FIG. 10: The ceiling in the Senate Chamber is an example of LOD 1.5.

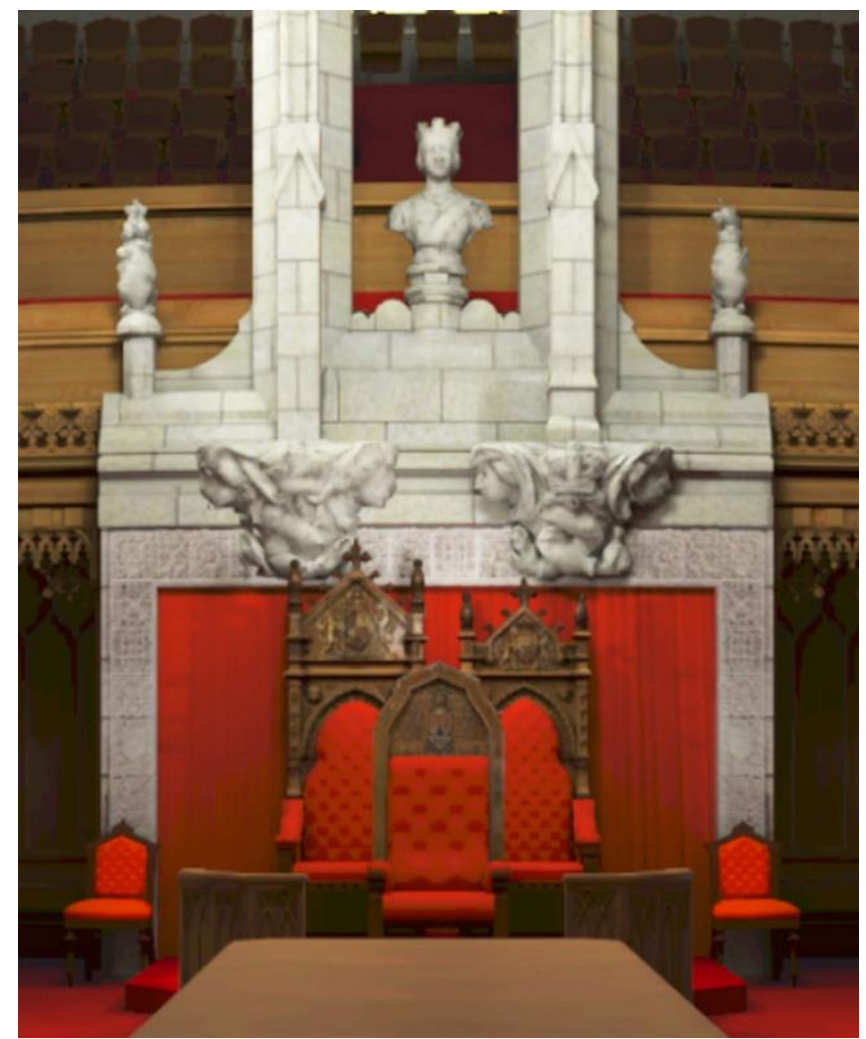

FIG. 11: The statues above the Speaker's chair in the Senate Chamber is an example of LOD 2.

\subsubsection{LOD 2}

This third and final category of the LOD system for heritage spaces in VR offers highly detailed geometry and photo-realistic colour. LOD 2 is a textured mesh that is created directly from metric data recorded with laser scanning or photogrammetry. It is, however, labour intensive and results in a higher polygon count. Even if steps are taken to reduce the polygon count through optimization, current technology will not support a virtual environment of the scale of this project composed entirely of LOD 2 elements due to polygon count and load times. The Centre Block VR project also had a tight schedule and scope, not allowing for the increased processing time 
of the meshes. The LOD 2 elements are highly detailed - using a combination of complex meshes, normal maps, and textures created from photogrammetry or laser scanning. LOD 2 heritage assets can be examined in close detail with a full sense of their materiality and geometry. LOD 2 elements are used for assets that are of the highest significance in the space. Through limiting their use, unique LOD 2 assets will draw the attention of the VR visitor, helping drive the narrative through the varying LODs.

Multiple LOD 2 items were developed for the Senate Chamber, including the wooden cherubs adorning the panelling, the stone aediculae along the wall, and the statues above the Speaker's chair platform (Figure 11). Each element was created through the processing of the photogrammetric model into a lower polygon mesh using InstaLOD. The mesh was textured using normal maps, bump maps, and UV maps to give the impression of greater depth and detail than the mesh contains.

\subsection{PROXIMITY BASED LOD}

1.1.1 For the Centre Block VR project, movement within the scenes is limited to how far the virtual visitor can physically walk in the space with no possibility of teleportation or joystick movement. This provided the opportunity to model elements farther away from the set point of view at a lower polycount. A careful selection of which assets should be modelled at higher LODs was critical. Prior to the commencement of each VR space, the project stakeholders would review the architectural qualities and cultural heritage value of the rooms, the narrative of the VR scene, and the positioning of the point of view of the viewer to help identify the desired LOD levels for each asset. LOD 1.5 and LOD 2 items were identified, and modelling commenced.

1.1.2 The treatment of all LOD assets was then determined by their distance to the point of view, using a proximity-based LOD. Elements that were close to the camera maintained a high-polycount, while elements at a distance, regardless of their LOD, were reduced to a lower-polycount mesh through the use of normal maps.

Normal maps create the illusion of depth on a flat surface by using RGB information in a texture layer of the object to indicate how the flat mesh surface should be shaded. The illusion of depth is created by the flat surface appearing to have the shadows of a more complex object. To create normal maps, a high and low polycount mesh of the same object are created in Rhinoceros 3D. The high-polycount mesh is used to generate the normal map's details in Autodesk 3DS Max, which are then projected onto the low-polycount mesh as a texture layer. The low-polycount mesh appears to have the details of the higher version when looked at from a distance; however, with close inspection, the illusion is broken.

Normal maps are efficient at reducing the polycount within a space by replacing the need for complex meshes with textures, as evident in the ornate wall detailing in the Senate Chamber (Figure 12). Unfortunately, normal maps are very time-consuming to create due to the need to model two separate versions of the meshes. This issue, in addition to their ineffectiveness when close to the point of view, warranted careful selection of when normal maps would be used.

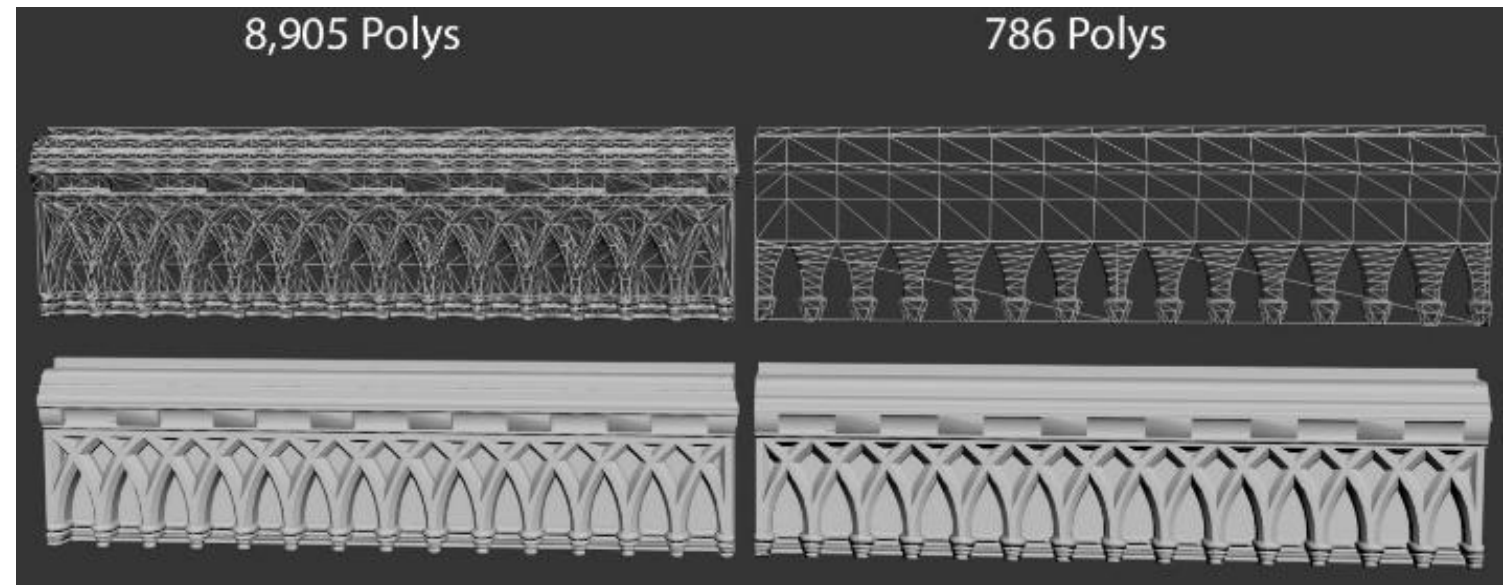

FIG. 12: Comparison in polycount between the mesh of the wall ornament in the Senate Chamber with and without normal maps. 
Within the Senate Chamber, the rows of desks were a taxing element on the polycount budget in the space. To reduce the burden of these assets, normal maps were intended to be used for all desks; however, the desks closest to the point of view were distracting at the low-polycount. To remedy the situation, three variations of the desks were created with different degrees of model complexity. Desks closest to the point of view contained all modelled details with no normal maps (Figure 9). With the virtual visitor able to walk up and look at the desks closely, it was important to ensure that the ornately carved frontages were three-dimensional. The desks farthest away were reduced to a simple box with little modelled detail. All wood detailing was included as an applied normal map. Desks in the mid-range contained a mixture of normal maps and modelled detail to create a gradual transition from high to low polycount desks (Figure 13).
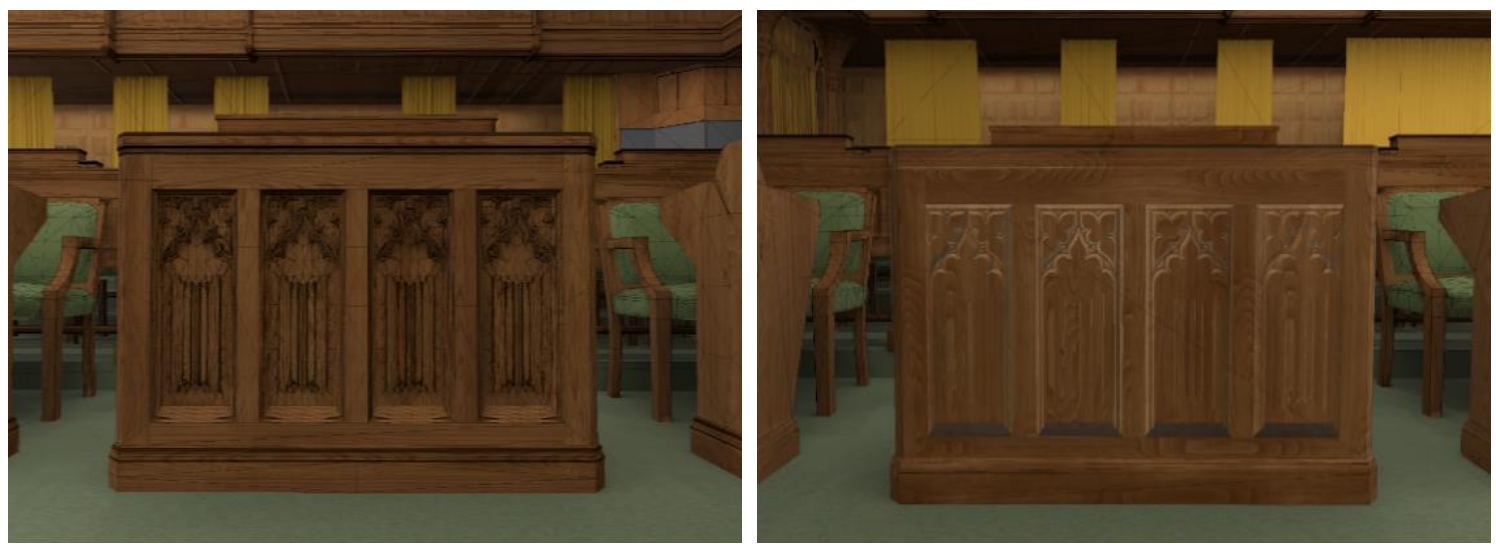

FIG. 13: Comparison between the Senate desks without and with normal maps.

Normal maps were crucial for LOD 2 elements. This was also the case for assets set further from the viewer. The sculptures above the Senate Speaker's chair platform were of high significance to the cultural heritage value of the space; however, they were at some distance from the point of view. To reduce the burden on the polycount budget within the space, they were reduced to a lower polygon count with normal maps. As seen in Figure 11, the facial detail of the sculptures is not well-defined, but adequate when viewing from across the room.

\section{LESSONS LEARNED}

The project proceeded by translating spaces from BIM to VR sequentially as requested by our project partners. This allowed for considerable development of the methodology by the completion of the final space, the Rotunda. The Rotunda would prove challenging to reduce to an adequate polygon count to meet the Oculus Rift VR requirements due to the geometric complexity of ornate stonework. Through examining the feedback from the previous spaces, the concept of proximity became an increased concern when making decisions for the reduction of meshes and using normal maps.

When approaching the Rotunda (Figure 14), the decision was made that no normal maps would be used for elements in the central circle with the point of view proposed to be directly beside the central column. Normal maps were only added in the central circle to increase the shadow detail within the ornate ribbing on the ceiling of this space. To direct attention to the end of the Hall of Honour that extended out of the Rotunda, the far wall was increased in detail with normal maps while the side walls remained reduced in visual stimuli. This aided in drawing attention to the areas of significance - like the entry to the Library of Parliament at the end of the Hall of Honour. The treatment of LOD 1.5 and LOD 2 items remained the same with the fauna and floral carvings in the stone frieze around the arches of the Rotunda and the central column at LOD 2 with the floor as the sole LOD 1.5 element. The LOD 2 of the fauna and flora varied in density to isolate elements to draw attention to, such as a bird or crest.

The approach to the Rotunda addressed the concerns with the proximity that arose through the development of the other spaces. Although LOD 1 elements are used in areas close and at a distance, their treatment is revised based on their proximity in addition to their significance. The result is a combination of the LODIA approach of the BIM highlighting areas of high cultural heritage value and the LOD approach of computer graphics relating solely to proximity. 


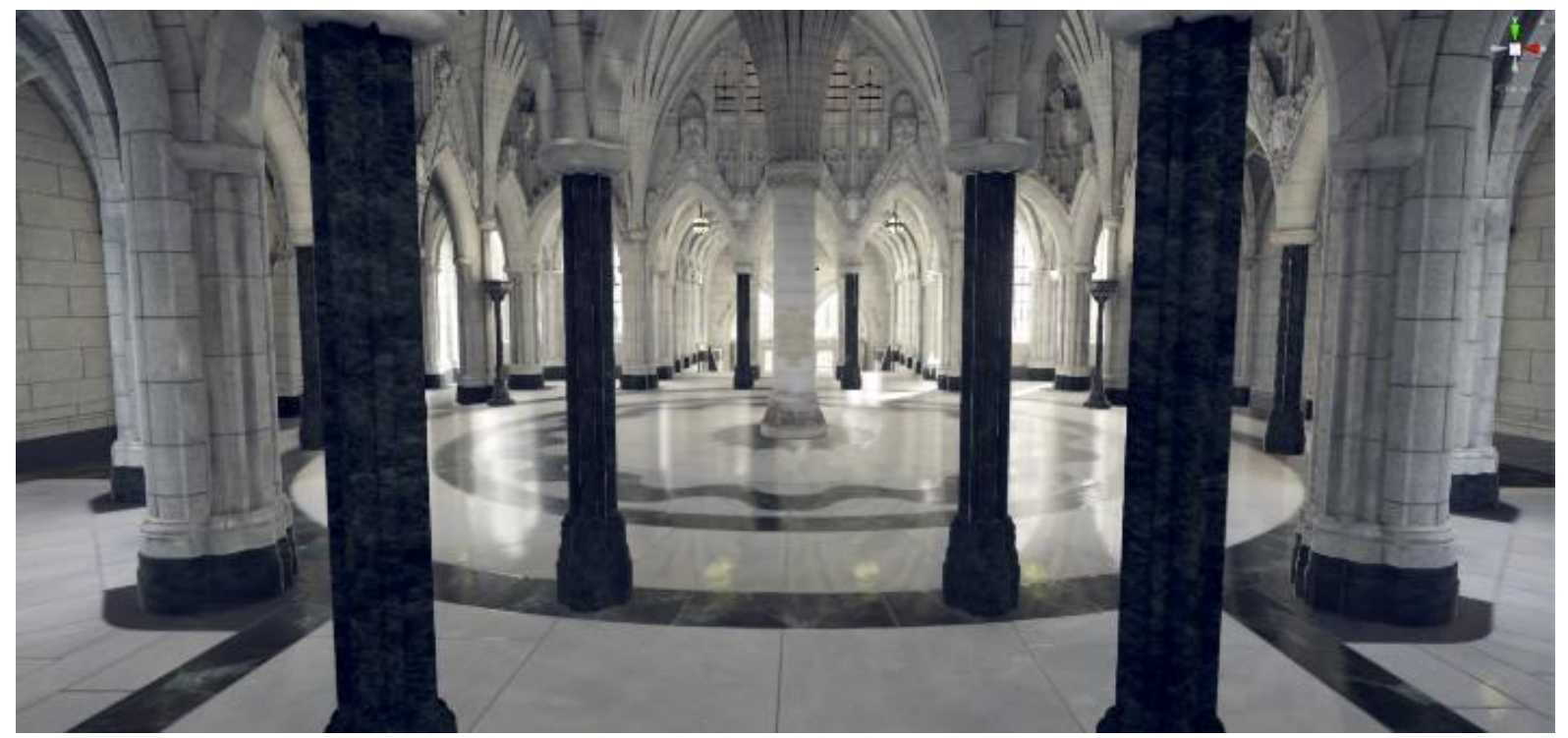

FIG. 14: Model in Unity 3D of the Rotunda.

\section{CONCLUSION}

Within virtual worlds, careful attention must be spent on ensuring a reduced polygon count for optimal processing speed and high fidelity. Level of detail techniques have been created to help define methods for reducing mesh complexities based on proximity. Discreet, continuous, and view-dependent methods have allowed for an object to appear in high detail when in close range and significantly reduced when at a distance. This technique, although transferable to the representation of heritage spaces, does not help manage the representation of large quantities of ornate detailing in a space that may all be seen at an equal distance. To help define a new LOD for the representation of heritage spaces in VR, the example of a BIM categorization system created by CIMS called the CIMS LODIA can be used. This system applies a higher value to elements of high cultural heritage value, providing them with a higher LOD. In the virtual experience, the elements of high cultural heritage value should be applied at a higher LOD than those not as important.

The division of assets within the space to the three LODs based on cultural heritage value helps maintain control over the polycount of the space and the scope and time commitment of its development. As LOD increases, the meshes become more complex and the processing of geometry is increased. With the limiting of a few objects in each space to LOD 2, determined by a review of the space by the project stakeholders, the final result was more congruent with the desired polycount and time commitment.

The LOD for VR of heritage spaces is not meant to be implemented independently of the existing LOD systems for computer graphics. The proximity-based LOD system proved successful when implemented with the LOD for VR heritage spaces to help determine when normal maps could be used to reduce polycount. By carefully applying normal maps to areas far from the point of view, the completed project maintained a balance between visual quality and computer performance. For the conversion of the Centre Block BIM into VR, the intended experience will not allow free movement through teleportation or joystick control, eliminating the need for discreet, continuous, or view-dependent LOD systems. For an experience with a greater degree of agency, these methods can be implemented for further control. 


\section{REFERENCES}

Antonopoulou, S., Bryan, P., (2017). BIM for Heritage: Developing a Historic Building Information Model. Historic England, Swindon, Historic England.

BIMFORUM, (2015). BIM Forum Level of Development (LOD) Specifications 2015.

BSI Group, (2013). PAS 1192-2:2013 Specification for Information Management for the Capital/Delivery Phase of Construction Projects using Building Information Modelling.

Clark, J., (1976). Hierarchical geometric models for visible-surface algorithms. Communications of the ACM 19, 547-554.

Dore, M.C., (2017). Current state of the art historic building information modelling. 42, 185-192.

Graham, K., Chow, L., Fai, S., (2018). Level of detail, information and accuracy in building information modelling of existing and heritage buildings. Journal of Cultural Heritage Management and Sustainable Development 8, 495-507.

Held, R.M., Durlach, N.I., (1992). Telepresence. Presence: Teleoperators and Virtual Environments 1, $109-112$. https://doi.org/10.1162/pres.1992.1.1.109

Hichri, N., Stefani, C., Luca, L., Veron, P., (2013). Review of the As-Built BIM Approaches, in: Nternational Archives of the Photogrammetry, Remote Sensing and Spatial Information Sciences - ISPRS Archives. Presented at the 5th International Workshop, 3D-ARCH-2013, Trento, Italy, pp. 107-112.

Luebke, D.P., (2003). Level of detail for 3D graphics. Morgan Kaufmann, Amsterdam.

Minsky, M., (1980). Telepresence. Omni 45-51.

Murray, J.H., (1997). Hamlet on the holodeck: the future of narrative in cyberspace. Free Press, New York.

Oculus Documentation, n.d. Guidelines for VR Performance Optimization [WWW Document]. URL https://developer.oculus.com/documentation/pcsdk/latest/concepts/dg-performance-guidelines/ (accessed 1.11.19).

Plowman Craven, (2015). BIM Survey Specification and Reference Guide. Plowman Craven, England.

Pybus, C., Graham, K., Doherty, J., Arellano, N., Fai, S., (2019). New Realities For Canada's Parliament: A Workflow For Preparing Heritage Bim For Game Engines And Virtual Reality. Presented at the CIPA 20019 Avilla 27th International Symposium, Avila, Spain.

Sheridan, T.B., (1992). Musings on Telepresence and Virtual Presence. Presence: Teleoperators \& Virtual Environments 1, 120-126. https://doi.org/10.1162/pres.1992.1.1.120

Slater, M., Usoh, M., (1993). An Experimental Exploration of Presence in Virtual Environments. Department of Computer Science Technical Reports: up to 1999.

Slater, M., Usoh, M., Steed, A., (1994). Depth of Presence in Virtual Environments. Presence 3, $130-144$. https://doi.org/10.1162/pres.1994.3.2.130

Steuer, J., (1992). Defining Virtual Reality: Dimensions Determining Telepresence. Journal of Communication 42, 73-93. https://doi.org/10.1111/j.1460-2466.1992.tb00812.x

Volk, R., Stengel, J., Schultmann, F., (2014). Building Information Modeling (BIM) for existing buildings Literature review and future needs. Automation in Construction 38, 109-127.

Zeltzer, D., (1992). Autonomy, Interaction, and Presence. Presence: Teleoper. Virtual Environ. 1, $127-132$. https://doi.org/10.1162/pres.1992.1.1.127 